\title{
DYNAMICAL MODEL OF THE ASYMMETRIC ACTUATOR OF DIRECTIONAL MOTION BASED ON POWER-LAW GRADED MATERIALS
}

\author{
Iakov A. Lyashenko ${ }^{1,2}$, Vadym N. Borysiuk ${ }^{2}$, Valentin L. Popov ${ }^{1,3,4}$ \\ ${ }^{1}$ Berlin University of Technology, Germany \\ ${ }^{2}$ Sumy State University, Ukraine \\ ${ }^{3}$ National Research Tomsk State University, Russia \\ ${ }^{4}$ Institute of Strength Physics and Materials Science, Tomsk, Russia
}

\begin{abstract}
We consider an actuator whose driving bodies are made of Power-Law graded materials. The directional motion is generated by an asymmetric mechanism producing simultaneously vertical and horizontal oscillations of the indenter. The dynamic contact of gradient materials is described and the equation of motion for the drive is written down and analyzed. It is shown that the exponent of the elastic inhomogeneity significantly affects the average velocity of motion of the cargo, which can be dragged by the drive.
\end{abstract}

Key Words: Piezoelectric Actuator, Friction, Method of Dimensionality Reduction, Normal and Tangential Contact Forces

\section{INTRODUCTION}

In modern robotics, electro-mechanical devices that convert electrical energy into mechanical energy are widely used to create directional motion. These devices can be realized as servo-drives [1], stepping motors [2], as well as piezoelectric drivers [3-5]. Piezoelectric actuators may be based on different principles; yet all of them have piezoelectric elements, which generate superimposed normal and horizontal oscillations. The phase shift between these oscillations defines the modes of produced motion. Such drives generate directional movement without an additional transmission mechanism in the system. However, if the actuator includes an asymmetric mechanism it is possible to

Received January 29, 2020 / Accepted March 30, 2020

Corresponding author: Iakov A. Lyashenko

Institute of Mechanics, Berlin Institute of Technology, Strasse des 17. Juni 135, 10623 Berlin, Germany

E-mail: i.liashenko@tu-berlin.de 
create directional motion using single source that produces oscillations only in the vertical direction $[3,4,6]$. Due to the asymmetry of the structure transmitting force from the piezoelectric element to the indenter, an additional tangential force occurs in the contact, which leads to the appearance of directional motion $[3,4,6]$.

To produce repeating stationary directional motion, it is necessary to keep a certain ratio between normal and tangential forces, as this parameter strongly affects the characteristics of produced movement. Such actuator of directional motion is a complex dynamic system, and its behavior is strongly affected by the inertial properties (mass of the cargo, moved by the drive) and the friction coefficient between the contacting elements. The elastic properties of the contacting materials and the geometrical shape of the indenter are also very important since these parameters determine the contact properties, and, therefore, the relationship between the normal and tangential forces. There are various methods for theoretical research and modeling of the above-described systems [7-9]. Within modern simulation methods the components of the forces (internal parameters), and the trajectory of the indenter, shifted by these forces, can be determined if the geometric sizes and elastic characteristics of the indenter are known. However, if we already know the indenter trajectory, it is possible to use more efficient and faster methods of numerical calculation. Thus, varying many different trajectories of the indenter in both directions, one can choose the optimal path which provides the necessary type of movement.

In general, the materials with graded elastic properties have found practical application in engineering as cutting tools, engine components, machine parts and other various devices [10]. There are several methods of obtaining of graded materials. One of them is coating as there are always graded properties at the boundary between different materials $[10,11]$. The main purpose of using mentioned materials is that in some conditions they exhibit alternative behavior, comparing to homogenous materials, and may somehow improve the properties of certain device of mechanism. In our previous study [12] we considered the behavior of the driving device parts which are made of homogenous materials. Thus, knowing that the approach implemented in [12] can be generalized to a similar system with power-law graded materials, it is natural to investigate its behavior and examine its operations mode. Another aim of our study is to check if the use of graded materials as contacting parts of the device can lead to any improvement or alternative behavior of the investigated system.

In [12] we proposed the dynamic model of a piezoelectric drive, which allowed us to simulate the movement of a cargo (or a slider) from already known indenter trajectories in both directions. In the framework of the proposed approach, we have performed the simulation of the drive experimentally studied in [3]. In the present work, we generalize the approach developed in [12] to power-law graded materials. When the slider of the actuator is made of power-law graded material, it is possible to tune the motion mode more precisely, which opens up new possibilities in robotics.

\section{NUMERICAL PROCEDURE}

As in the previous work [12], for modeling phenomena we shall use the method of dimensionality reduction (MDR), the framework of which, in the case of power-law graded 
materials, is described in detail in [13]. We consider the case where the shear modulus of a power-law graded material $E$ depends on normal coordinate $z$ according to:

$$
E(z)=E_{0}\left(z / c_{0}\right)^{k},
$$

where exponent of elastic inhomogeneity $k$ varies in the range $0 \leq k<1$.

The MDR approach allows finding exact solution for three-dimensional problem of a contact of axially symmetric bodies by mapping it onto an equivalent one-dimensional model. If both contacting bodies are made of the same material (as is the case in our study), the normal and tangential contact problems are decoupled [13, 14] and can be considered independently within the framework of MDR as well. Let us consider the contact of an axially symmetric elastic object characterized by three-dimensional profile $z=f(r)$ with elastic half-space. Equivalent one-dimensional profile $g(x)$ defined as $[13,15]$ :

$$
g(x)=|x| \int_{0}^{|x|} \frac{f^{\prime}(r)}{\sqrt{\left(x^{2}-r^{2}\right)^{1-k}}} \mathrm{~d} r .
$$

For simplicity let us consider parabolic indenter $f(r)=r^{2} /(2 R)$ with radius $R$, in this case $g(x)$ according to Eq. (2) can be written as:

$$
g(x)=\frac{x^{2}}{(k+1) R} .
$$

When $g(x)$ is known, the next step is to replace the elastic half-space by the Winkler elastic foundation: a linear array of non-interacting springs with normal and tangential rigidities:

$$
\begin{aligned}
& k_{z}(x)=c_{N}(x) \Delta x=\left(\frac{1-\nu_{1}^{2}}{h_{N}\left(k, \nu_{1}\right) E_{01}}+\frac{1-\nu_{2}^{2}}{h_{N}\left(k, \nu_{2}\right) E_{02}}\right)^{-1}\left(\frac{|x|}{c_{0}}\right)^{k} \Delta x, \\
& k_{x}(x)=c_{T}(x) \Delta x=\left(\frac{1}{h_{T}\left(k, \nu_{1}\right) E_{01}}+\frac{1}{h_{T}\left(k, \nu_{2}\right) E_{02}}\right)^{-1}\left(\frac{|x|}{c_{0}}\right)^{k} \Delta x,
\end{aligned}
$$

where functions $h_{N}(k, v)$ and $h_{T}(k, v)$ defined in open access literature [13] (see page 267 for exact definition), so we are not writing their exact form here. In Eqs. (3) and (4) $\Delta x$ is the step of space discretization (distance between springs), and $v_{1,2}$ are Poisson's ratios of the indenter and half-space materials. Elastic parameters $E_{01}$ and $E_{02}$ for indenter and half-space are also introduced (see Eq. (1)). As mentioned before, we consider the situation where the indenter and the slider are made of the same material i.e. $E_{01}=E_{02}=E_{0}$ and $v_{1}=v_{2}=v$. In this case, instead of Eqs. (3) and (4) we can use simplified expressions:

$$
k_{z}(x)=\frac{h_{N}(k, \nu) E_{0}}{2\left(1-\nu^{2}\right)}\left(\frac{|x|}{c_{0}}\right)^{k} \Delta x, \quad k_{x}(x)=\frac{1}{2} h_{T}(k, \nu) E_{0}\left(\frac{|x|}{c_{0}}\right)^{k} \Delta x,
$$


In the next step, one-dimensional profile $g(x)$ is indented into the Winkler foundation. If the indentation depth (position of the indenter) is $d$ then the displacement of the individual springs in the contact can be obtained as

$$
u_{z}(x)=d-g(x)=d-\frac{x^{2}}{(k+1) R} .
$$

Denoting the radius of contact by $a$, we write $u_{\mathrm{z}}(a)=0$, and resolve Eq. (6) with respect to $d$ :

$$
d=\frac{a^{2}}{(k+1) R} .
$$

Total normal force $F_{z}$ in the contact area is determined by the sum of the contributions of forces from all the compressed springs (we consider the case without adhesion) in the contact:

$$
F_{z}(a)=\int_{-a}^{a} c_{N}(x) \cdot u_{z}(x) \mathrm{d} x=\frac{2 E_{0}}{\left(1-\nu^{2}\right)} \frac{h_{N}(k, \nu) a^{3+k}}{c_{0}^{k}(k+1)^{2}(k+3) R} .
$$

Simulation of the dynamic tangential contact within the MDR can be performed as follows. We first assume that all the springs that are in contact with the indenter are displaced along with the indenter during the tangential motion. We denote the tangential displacements of the individual springs as $u_{x}\left(x_{i}\right)$. In each iteration the condition

$$
\mu c_{N}(x) u_{z}\left(x_{i}\right) \leq\left|c_{T} u_{x}\left(x_{i}\right)\right|
$$

is checked, where $\mu$ is the friction coefficient. It can be re-written as

$$
\mu \frac{h_{N}(k, \nu)}{\left(1-\nu^{2}\right)} u_{z}\left(x_{i}\right) \leq h_{T}(k, \nu)\left|u_{x}\left(x_{i}\right)\right| .
$$

For the springs which fulfill this condition, a new value of displacements should be set:

$$
u_{x}\left(x_{i}\right)= \pm \mu \frac{h_{N}(k, \nu) u_{z}\left(x_{i}\right)}{h_{T}(k, \nu)\left(1-\nu^{2}\right)},
$$

where the sign is the same as the sign of $u_{x}\left(x_{i}\right)$ prior to the updating of its value. After this, tangential force (friction force) $F_{x}$ is calculated through the equation:

$$
F_{x}(a)=2 \int_{0}^{a} c_{T}(x) u_{x}(x) \mathrm{d} x=\frac{E_{0} h_{T}(k, \nu)}{c_{0}^{k}} \int_{0}^{a}|x|^{k} u_{x}(x) \mathrm{d} x .
$$

For discretization of the numerical model, integrals (8) and (12) are replaced by the corresponding sums:

$$
F_{z}=\sum_{\text {cont }} k_{z}(x) \cdot u_{z}\left(x_{i}\right), \quad F_{x}=\sum_{\text {cont }} k_{x}(x) \cdot u_{x}\left(x_{i}\right),
$$

where the sum is taken over all the springs in contact. 


\section{DYNAMIC MODEL OF THE MECHANIC DRIVER}

Fig. 1 shows a schematic presentation of the actuator that was experimentally studied in [3]. An analogous system with the slider made of homogeneous material was theoretically studied in [12]. Here the slider with mass $M$ is placed on a hard-fixed base. The friction coefficient in the contact between the slider and the base equals $\mu_{1}$. The slider is moved by a parabolic indenter, the friction coefficient in the indenter-slider contact equals $\mu$. The indenter and the slider are made of material with elastic modulus $E(1)$, and Poisson ratio $v$.

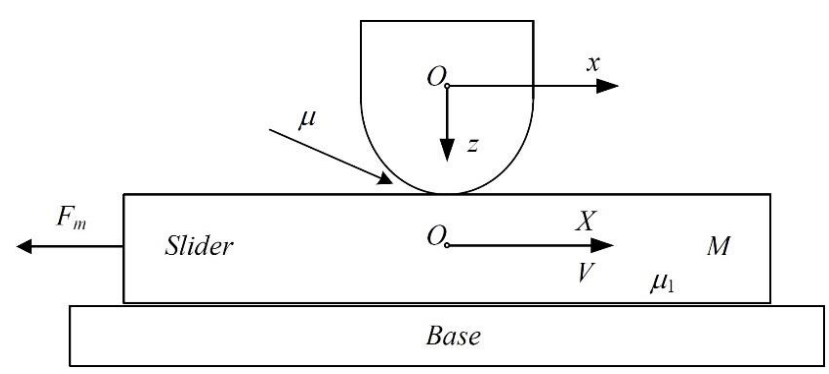

Fig. 1 Schematic presentation of the driver

In Fig. 1, horizontal and vertical coordinates of the indenter are denoted $x$ and $z$, respectively. The indenter movement causes horizontal shift of slider $X$. The slider located on the fixed base did not move in vertical direction.

In this work, we consider the ability of the driver to resist external force $F_{m}$, which is applied against the direction of movement. Therefore, our aim is to find the critical value of constant returning force $F_{m}$ at which the drive is still capable to shift the slider in positive direction $O X$. We consider the situation with an already known trajectory of an indenter (see Fig. 1). In this simplified case, dynamical model for the slider movement consists of a single equation of motion [12]:

$$
M \ddot{X}=F_{x}-F_{b a s e}-F_{m},
$$

where $F_{x}$ is tangential friction force between the indenter and the slider, calculated with Eq. (13). The friction force in Eq. (14) is written with the "+" sign since it is the driving force that causes movement of the slider. As the slider moves, there is a friction force $F_{\text {base }}$ :

$$
F_{\text {base }}=\operatorname{sgn}(\dot{X}) \mu_{1} F_{z}
$$

In Eq. (15), normal force $F_{z}$ calculated with Eq. (13). Note that to take into account the direction of the acting force in Eq. (15) we use standard sign function $\operatorname{sgn}(\dot{X})$, with $\dot{X} \equiv V$, where $V$ is velocity of the slider.

One of the most important features of the numerical model is correct determination of the displacement of the springs according to Eqs. (9) - (11). Here, to check the sliding criterion at each step, the indenter displacement must be added incrementally to the horizontal displacement of springs, and condition (9) must be checked for all springs. However, we consider the case where the slider can move simultaneously with the indenter. Therefore, instead of displacement of indenter $x$, the relative displacement of 
the indenter against slider $\Delta \tilde{x}$ should be added to the elongation of the springs at each step. The mentioned displacement of the indenter can be calculated through the equation

$$
\Delta \tilde{x}_{i}=\left[x\left(t_{i}+\Delta t\right)-x\left(t_{i}\right)\right]-\left[X\left(t_{i}+\Delta t\right)-X\left(t_{i}\right)\right],
$$

where $t_{i}$ is incrementally increasing time, $\Delta t$ is the step of numerical integration of Eq. (14).

To investigate the operating modes of the considered directional drive, we need to set the trajectory of the indenter shown in Fig. 1. As such a trajectory, we shall use, analogously to [12], the dependences obtained from the experimental results in [3]. Thus, the trajectory of the indenter can be described by a linear dependence as:

$$
\begin{gathered}
N=\text { integer }\left(\frac{t}{t_{1}+t_{2}}\right), \\
A=\left\{\begin{array}{ll}
\frac{A_{\max }\left[t-N\left(t_{1}+t_{2}\right)\right]}{t_{1}}, & \text { if } \quad N\left(t_{1}+t_{2}\right) \leq t \leq N\left(t_{1}+t_{2}\right)+t_{1} \\
A_{\max } \frac{(N+1)\left(t_{1}+t_{2}\right)-t}{t_{2}}, & \text { otherwise }
\end{array},\right.
\end{gathered}
$$

where $A_{\max }$ is an amplitude, $t$ is time, $N$ is an integer and integer(.) is the integer division operation, indicating the period number. Usually, in the experiments, the normal oscillation amplitude of the indenter is substantially smaller than the tangential amplitude. Thus, we consider the time dependencies of indenter coordinates $x(t)$ and $z(t)$, obtained earlier in [12] that are shown in Fig. 2.

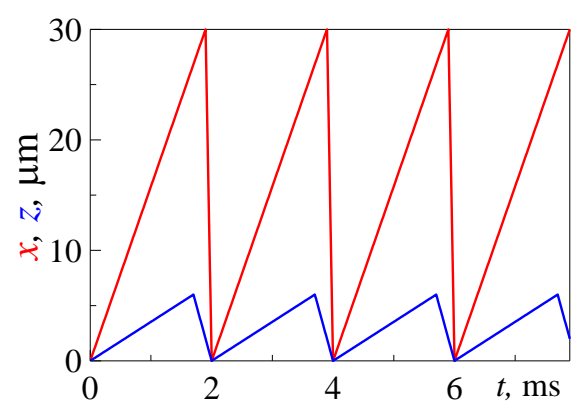

Fig. 2 Time dependencies of the indenter coordinate, shown in Fig. 1. Dependencies obtained from the Eqs. (17) and (18) at parameters $A_{\max }=30 \mu \mathrm{m}, t_{1}=1.9 \mathrm{~ms}, t_{2}=0.1$ ms for $x(t)$ and $A_{\max }=6 \mu \mathrm{m}, t_{1}=1.7 \mathrm{~ms}, t_{2}=0.3 \mathrm{~ms}$ for $z(t)$.

We shall use a set of parameters as in our previous work [12]: indenter radius $R=2.5$ $\mathrm{mm}$, the friction coefficient between the surfaces of the indenter and the slider $\mu=0.3$, the friction coefficient between the surfaces of the indenter and the base $\mu_{1}=0.1$, slider mass $M=50 \mathrm{~g}$. Previously we considered the case of the homogeneous materials with elastic parameters $E=2 \cdot 10^{11} \mathrm{~Pa}$ and $v=0.3$ [12], which correspond to steel. Here we 
consider the case where the elastic modulus of the indenter and the slider materials $E$ depends on the indentation depth according to Eq. (1).

In further simulations we will set $E_{0}=2 \cdot 10^{11} \mathrm{~Pa}$ and $v=0.3$. Then, at $k=0$ we must arrive at the results, related to homogeneous materials, obtained in [12]. However, in [12] effective parameter for contact of homogeneous materials $E^{*}=0.5 /\left(1-v^{2}\right)$ was mistakenly determined without coefficient 0.5. In [12] we used constant values of effective elastic modulus $E^{*}$ and effective shear modulus $G^{*}$. From the relations between $E^{*}$ and $G^{*}$, it is easy to see that the simulation performed in [12] corresponds to the set of parameters $E=1.9838 \cdot 10^{11} \mathrm{~Pa}$ and $v=0.74076$. Such magnitude of Poisson ratio is not physically relevant, as for thermodynamically stable materials, Poisson ratio $v$ does not exceed 0.5 [16]. So in proposed work we will consider contact of homogenous materials with $k=0$ as an additional specific case because in the case $k=0$ we will have the situation $E_{0}=2 \cdot 10^{11} \mathrm{~Pa}$ and $v=0.3$, how it should have been in [12]. Results of simulations for $k=0$ shown in Fig. 3.

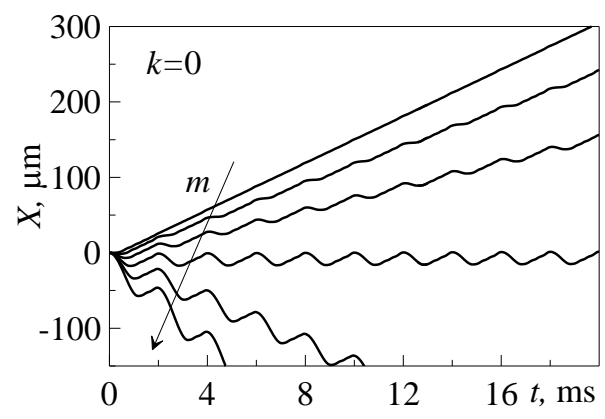

Fig. 3 Time dependencies of the coordinate $X$ of the slider, shown in Fig. 1. Mass of the cargo $m$ varies from 0 to $1.0 \mathrm{~kg}$ with increment $0.2 \mathrm{~kg}$, mass increasing is shown with arrow

For numerical solution of the Eq. (14) we use the Euler method with an integration time step $\Delta t=10^{-7} \mathrm{~s}$. The discretization step on coordinate (distance between the springs in the MDR method) was set as $\Delta x=10^{-8} \mathrm{~m}$.

Fig. 3, as in our previous work [12], presents the movement for varying mass of a cargo moved by the driver shown in Fig. 1. The driver performs the work against the force field, where the force, acting against motion defined as gravity force $F_{m}=m g$. It can be seen that the driver performs directional movement of the load, providing a positive average speed of the slider, provided the mass of the load does not exceed a certain critical value. It also follows from the figure that there are two modes of the driver operation: moving the slider in one direction (upper curves), and stick-slip mode of movement in which the slider velocity periodically changes its sign. In stick-slip mode it is important for the driver to provide a positive average velocity during one period, in this case it will continue to perform the work at moving cargo. Next, we determine this average velocity, which depends on the mass of the cargo and the exponent of the elastic inhomogeneity of the material from which the slider and the indenter are made.

From Eq. (1), it follows that for $c_{0}>1 \mathrm{~m}$ and $c_{0}<1 \mathrm{~m}$ increasing (or decreasing) value of exponent $k$ affects the behavior of the studied system in different ways (due to 
the presence of factor $c_{0}^{k}$ ). In this work, we will not consider these features, and will use value $c_{0}=1 \mathrm{~m}$.

Results of the performed simulation at different values of the exponent of the elastic inhomogeneity $k$ are shown in Fig. 4. Left panel of the figure shows the trajectories of the slider at the cargo mass $m=0.1 \mathrm{~kg}$ and three values of exponent $k$.
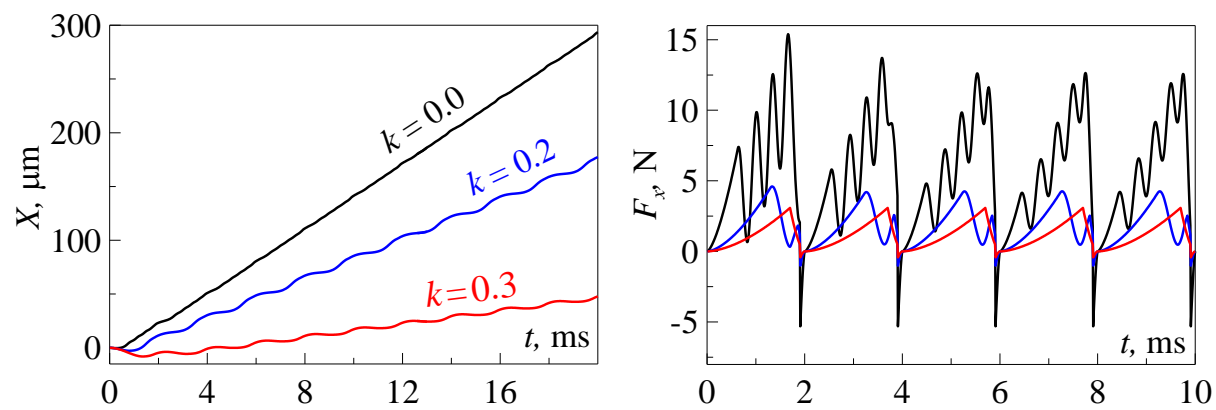

Fig. 4 (left panel) Time dependence of the slider coordinate $X$ shown in Fig. 1 at cargo mass $m=0.1 \mathrm{~kg}$, and different values of the exponent $k$ (shown in figure); (right panel) Time dependencies of the tangential forces, acting in contact, related to dependencies in the left panel

As can be seen Fig.4, increasing of the exponent of the elastic inhomogeneity causes reduction of the efficiency of the device. As parameter $k$ increases, material becomes softer, while normal and tangential forces acting in contact decrease, which leads to mentioned above situation. For instance, the right panel of Fig. 4 shows time dependencies of tangential force with decreasing magnitude as the $k$ growth. However, in all studied cases $(k=0,0.2$ and 0.3$)$ the device works as the driver of directional motion, dragging the cargo against gravity force $F_{m}=m g$. This fact shows that power-law graded materials can be used for manufacturing of such mechanical devices. It is worth mentioning that, according to Fig. 4, all considered cases have a transient mode at the beginning of motion, followed by the stationary mode of directional motion after several periods.

In [12] we analyzed averaged over period velocity of the slider, depending on the cargo mass $m$, which define gravity force $F_{m}=m g$. In the case of power-law graded materials, to perform a similar analysis we need to take into account exponent of elastic inhomogeneity $k$, as it also affects the efficiency of the driver.

We assume that the device reaches the stationary operation mode after first 5 periods (see Fig. 4). Thus, to estimate average velocity we skipped the first 5 periods, and during the sixth period, the average velocities were calculated. Calculated dependencies of average velocity $\langle V\rangle$ of the slider on cargo mass and exponent $k$ are shown in Fig. 5. 

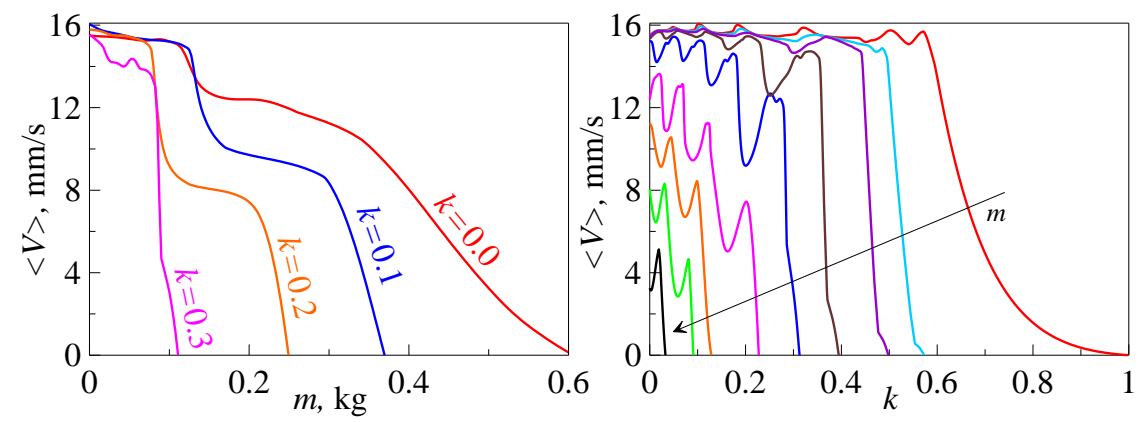

Fig. 5 (left panel) Dependencies of averaged over period velocity $\langle V\rangle$ of the slider on cargo mass $m$ at different values of the exponent $k$; (right panel) Dependencies of averaged over period velocity of the slider on exponent $k$ at different values of cargo mass $m=0 ; 0.01 ; 0.02 ; 0.05 ; 0.1 ; 0.2 ; 0.3 ; 0.4 ; 0.5$; increasing of mass is shown with arrow

One can see that with increasing of gradient exponent $k$ and cargo mass $m$, the driving velocity decreases. Therefore, we can conclude that to ensure high drive performance it is best to use homogeneous materials with a constant value of the elastic modulus (with $k=0$ ). However, the dependencies of average velocity on gradient exponent $k$ (right panel of the figure) are not monotonic. So, it is not clear if an increase of exponent $k$ leads to a decrease in the efficiency of the driver of directional motion. We examined the situation in which the indenter and the slider are made of the same power-law graded material. It is possible that in another situation (for example, a rigid indenter and a slider made of power-law graded material) it will be possible to achieve higher driver performance.

\section{CONCLUSIONS}

We developed a mathematical model of an actuator of directed motion with the driving parts made of power-law graded materials. We show that within the range of gradient exponent $0 \leq k<1$ the driver can produce the directed motion of the cargo, performing positive work. Moreover, although at first glance the situation of using homogeneous materials $(k=0)$ seems to be optimal, the dependence of the average slider velocity on the gradient parameter, is non-monotonous, which suggests that with different relations between the model parameters, a significant increase in drive efficiency may be achieved. In proposed work, we developed and described a model of the driver made of power-law graded materials; however, to clarify its effectiveness it is necessary to carry out further analysis with respect to additional parameters, which is not the purpose of this work. It is worth noting that another possible solution in the field of producing of the directed motion can be an interaction with a periodic relief created artificially or implemented at the molecular level $[17,18]$. 
Acknowledgments: This work was partially supported by the Fundamental Research Program of the State Academies of Sciences for 2013-2020, line of research III.23 and the Tomsk State University competitiveness improvement programme. VNB is grateful to the Ministry of Education and Science of Ukraine for financial support (Project No. 0117U003923).

\section{REFERENCES}

1. Ross, R., 2014, Investigation into soft-start techniques for driving servos, Mechatronics, 24(2), pp. 79-86.

2. Wawszczak, W., Towarek, Z., Jagiełł, B., 2004, Methodology of the stepper motor rotational motion investigations, Mechanics and Mechanical Engineering, 7(1), pp. 87-95.

3. Cheng, T.H., He, M., Li, H.Y., Lu, X.H., Zhao, H.W., Gao, H.B., 2017, A novel trapezoid-type stick-slip piezoelectric linear actuator using right circular flexure hinge mechanism, IEEE Transactions on Industrial Electronics, 64(7), pp. 5545-5552.

4. Nguyen, H.X., Teidelt, E., Popov V.L., Fatikow, S., 2016, Modeling and waveform optimization of stick-slip micro-drives using the method of dimensionality reduction, Archive of Applied Mechanics, 86, pp. 1771-1785.

5. Li, J., Zhao, H., Shao, M., Zhou, X., Fan, Z., 2015, Design and experimental research of an improved stick-slip type piezo-driven linear actuator, Advanced in Mechanical Engineering, 7(9), pp. 1-8.

6. Popov, V.L., 2017, Oscillation-based methods for actuation and manipulation of nano-objects, AIP Conference Proceedings, 1882, pp. 020056: 1-5.

7. Alshamasin, M.S., Ionescu, F., Al-Kasasbeh, R.T., 2012, Modelling and simulation of a SCARA robot using solid dynamics and verification by MATLAB/Simulink, International Journal of Modelling Identification and Control, 15(1), pp. 28-38.

8. Sui, L., Xiong, X., Shi, G., 2012, Piezoelectric actuator design and application on active vibration control, Physics Procedia, 25, pp. 1388-1396.

9. Kelemen, A., Crivii, M., Trifa, V., 1987, Mathematical modeling and simulation of stepping motor systems, Mathematical Modelling, 8, pp. 544-549.

10. Miyamoto, Y., Kaysser, W.A., Rabin, B.H., Kawasaki, A., Ford, R.G. (Eds.), 1999, Functionally graded materials: Design, processing and applications, Dordrecht: Kluwer Academic, $330 \mathrm{pp}$

11. Shugurov, A.R., Kuzminov, E.D., Kasterov, A.M., Panin, A.V., Dmitriev, A.I., 2020, Tuning of mechanical properties of $\operatorname{Ti}_{1-x} A l_{x} N$ coatings through Ta alloying, Surface and Coatings Technology, 382, pp. 125219.

12. Lyashenko, I.A., Borysiuk, V.N., Popov, V.L., 2019, Dynamical model of asymmetric actuator of directional motion, Meccanica, 54(10), pp. 1681-1687.

13. Hess, M., 2016, A simple method for solving adhesive and non-adhesive axisymmetric contact problems of elastically graded materials, International Journal of Engineering Science, 104, pp. 20-33

14. Popov V.L., Heß M., Willert E., 2019, Handbook of contact mechanics: exact solutions of axisymmetric contact problems, Berlin: Springer, $347 \mathrm{pp}$.

15. Willert, E., Dmitriev, A.I., Psakhie, S.G., Popov, V.L., 2019, Effect of elastic grading on fretting wear, Scientific Reports, 9, pp. 7791: 1-8.

16. Landau, L.D., Lifshitz, E.M., 1986, Theory of Elasticity, New York, Pergamon Press, 165 pp.

17. Popov, V.L., 2004, Nanomachines: a general approach to inducing directed motion at the atomic level, International Journal of Non-Linear Mechanics, 39(4), pp. 619-633.

18. Popov, V.L., 2003, Nanomachines: methods to induce a directed motion at nanoscale, Physical Review E, 68(2), 026608: 1-7. 